\title{
Susceptibility-Weighted MR Imaging: A Better Technique in the Detection of Capillary Telangiectasia Compared with T2* Gradient-Echo
}

\author{
U.S. Chaudhry, D.E. De Bruin, and B.A. Policeni
}

\begin{abstract}
BACKGROUND AND PURPOSE: Enhancing lesions on brain MR imaging can present a diagnostic quandary as both benign lesions such as brain capillary telangiectasia and pathologic lesions such as demyelination may appear similar. Stagnation of blood in low-flow venous channels of brain capillary telangiectasias results in susceptibility effect secondary to the increased local deoxyhemoglobin. Both $\mathrm{T2}$ * gradient-echo imaging and SWI were demonstrated as valuable in the diagnosis of brain capillary telangiectasia. Because SWI is more sensitive to susceptibility changes than gradient-echo, we aim to demonstrate increased diagnostic value of SWI compared with gradientecho in making the diagnosis of brain capillary telangiectasia.
\end{abstract}

MATERIALS AND METHODS: We retrospectively reviewed the MR images of 17 patients with a presumed diagnosis of brain capillary telangiectasia and who were examined from June 2010 to September 2012. All patients underwent MR imaging at 1.5T with T1, T2, FLAIR, gradient-echo, SWI, and gadolinium-enhanced T1 sequences. Lesions were evaluated for the presence or absence of signal abnormality on each particular sequence.

RESULTS: All 17 brain capillary telangiectasias demonstrated distinct signal-intensity loss on SWI compared with 7 of 17 (41\%) who showed signal-intensity loss on gradient-echo. The increased frequency of detection using SWI versus gradient-echo is statistically significant $(z=$ $2.85, P<.01 ; \chi^{2}=8.10, P<.01$ ). Six of the lesions showed signal-intensity changes on $\mathrm{T1}$ and/or $\mathrm{T} 2$ whereas the remaining lesions were isointense to normal brain.

CONCLUSIONS: Brain capillary telangiectasias are more conspicuous on SWI than gradient-echo imaging and other precontrast MR imaging. SWI is a valuable tool in diagnosing these benign lesions and should serve to increase diagnostic confidence.

ABBREVIATIONS: $\mathrm{BCT}=$ brain capillary telangiectasia; $\mathrm{GRE}=$ gradient-echo

B rain capillary telangiectasia (BCT) is considered the most benign vascular malformation in the brain. ${ }^{1}$ This is usually incidental as it is almost always asymptomatic. ${ }^{2}$ It is described as having an inconspicuous appearance on precontrast MR imaging with faint or brush-like enhancement on postcontrast imaging. ${ }^{3,4}$ Its importance lies in the fact that it may be confused with much more serious pathologies such as metastasis, acute demyelination, and subacute infarction. ${ }^{2}$ This issue is only going to become more of a challenge with increased quality of MR imaging and increasing field strengths, which will increase susceptibility effects. A few

Received March 28, 2014; accepted after revision May 23.

From the Department of Radiology (U.S.C., D.E.D., B.A.P.) and Section of Neuroradiology (D.E.D., B.A.P.), University of lowa Hospitals and Clinics, lowa City, lowa.

Paper previously presented as electronic poster at: Annual Meeting of the American Society of Neuroradiology, May 18-23, 2013; San Diego, California.

Please address correspondence to Bruno A. Policeni, MD, Department of Radiology, University of lowa Hospitals and Clinics, 200 Hawkins Dr, lowa City, IA; e-mail: bruno-policeni@uiowa.edu

http://dx.doi.org/10.3174/ajnr.A4082 previous publications have addressed utility of gradient-echo (GRE) and DWI for BCT diagnosis. ${ }^{3-5}$ SWI is a relatively new concept relying on susceptibility effect secondary to the increase in local deoxyhemoglobin. ${ }^{6}$ SWI has been shown to be more sensitive to magnetic field susceptibility than GRE. ${ }^{7}$ There does remain a need for a study to compare the sensitivity of SWI versus GRE images when it comes to diagnosing a BCT with certainty.

The purpose of the study was to demonstrate an increased diagnostic value of SWI compared with GRE in making the diagnosis of BCT. Comparison to other modalities such as T2weighted imaging and DWI were also made.

\section{MATERIALS AND METHODS}

Subjects were selected using a keyword search for "capillary telangiectasia" on all MR imaging radiology reports with a date range from June 1, 2011 (coinciding with the initial implementation of SWI on our MR scanners) to September 30, 2012. This yielded 209 studies. Individual studies were then reviewed in con- 
sensus by 2 board-certified radiologists, 1 with a Certificate of Added Qualification in neuroradiology. T1, T2, SWI, GRE, and postcontrast images were all reviewed to establish the diagnosis of BCT. A total of 17 patients with lesions meeting diagnostic criteria

Table 1: Comparison of detection of capillary telangiectasia among different sequences

\begin{tabular}{cc}
\hline Imaging Sequence & $\begin{array}{c}\text { No. of Patients } \\
\text { Detected }(\boldsymbol{n}=17)\end{array}$ \\
\hline T1 & $1(5.9 \%)$ \\
T2 & $6(35.3 \%)$ \\
FLAIR & $6(35.3 \%)$ \\
GRE & $7(41.2 \%)$ \\
SWI & $17(100 \%)$ \\
\hline
\end{tabular}

\section{Table 2: Distribution of BCT according to location in the study}

\begin{tabular}{lc}
\hline \multicolumn{1}{c}{ Location } & $\begin{array}{c}\text { No. of Patients } \\
\text { with BCT }(\boldsymbol{n}=17)\end{array}$ \\
\hline $\begin{array}{l}\text { Pons } \\
\text { Cerebral hemispheres, excluding gray } \\
\quad \text { matter nuclei }\end{array}$ & $8(47.0 \%)$ \\
Deep supratentorial gray matter nuclei & $6(35.3 \%)$ \\
Cerebellum & $1(5.9 \%)$ \\
Midbrain & $1(5.9 \%)$ \\
\hline
\end{tabular}

for BCT who also underwent SWI were found. No patients underwent biopsy or surgical resection of these BCTs.

Imaging was done on 1.5T MR scanners (Avanto; Siemens, Erlangen, Germany). All patients underwent standard MR imaging including axial spin-echo T1- and T2-weighted imaging, FLAIR, DWI, T2*-GRE, SWI, and postcontrast fat-saturated axial T1-weighted imaging. Intravenous gadobenate dimeglumine (MultiHance; Bracco Diagnostics, Princeton, New Jersey) was administered at a concentration of $0.1 \mathrm{mmol} / \mathrm{kg}$.

\section{RESULTS}

Seventeen lesions meeting diagnostic criteria for BCT were identified in 17 patients. All 17 BCTs demonstrated distinct signalintensity loss on SWI (Table 1). Figure 1-3 represent the spectrum of lesions seen, varying from some seen only on SWI, to some seen in both. Seven of the total 17 (41\%) demonstrated signal-intensity loss on GRE imaging. Six of the lesions showed signal-intensity changes on $\mathrm{T} 1$ and/or T2, whereas the remaining lesions were isointense to normal brain (Table 1). Most of the lesions were in the pons, $47 \%$ (Table 2).

A McNemar test for dependent proportions was used for statistical analysis. ${ }^{8}$ Increased frequency of detection by SWI
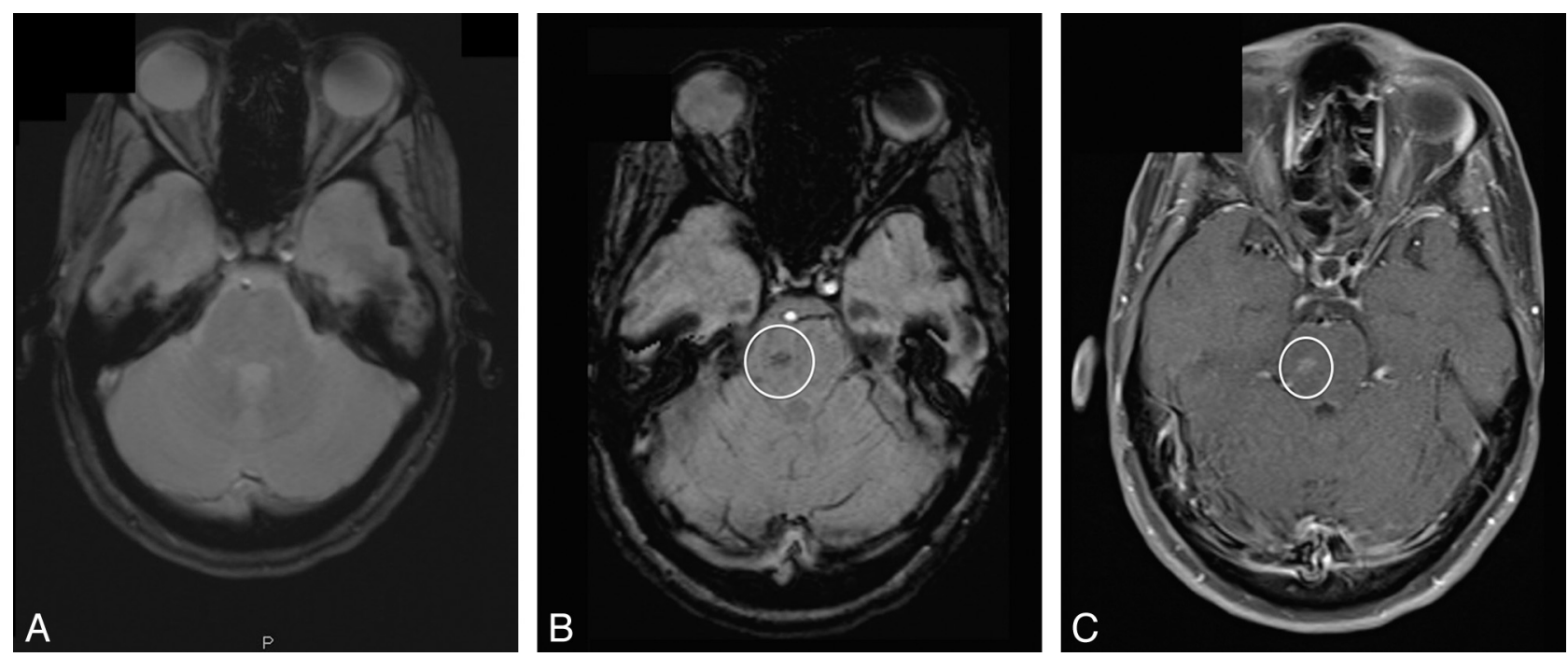

FIG 1. A BCT (circle) not seen on GRE image $(A)$ but seen on SWI $(B)$ and postcontrast $T 1$ image (C).
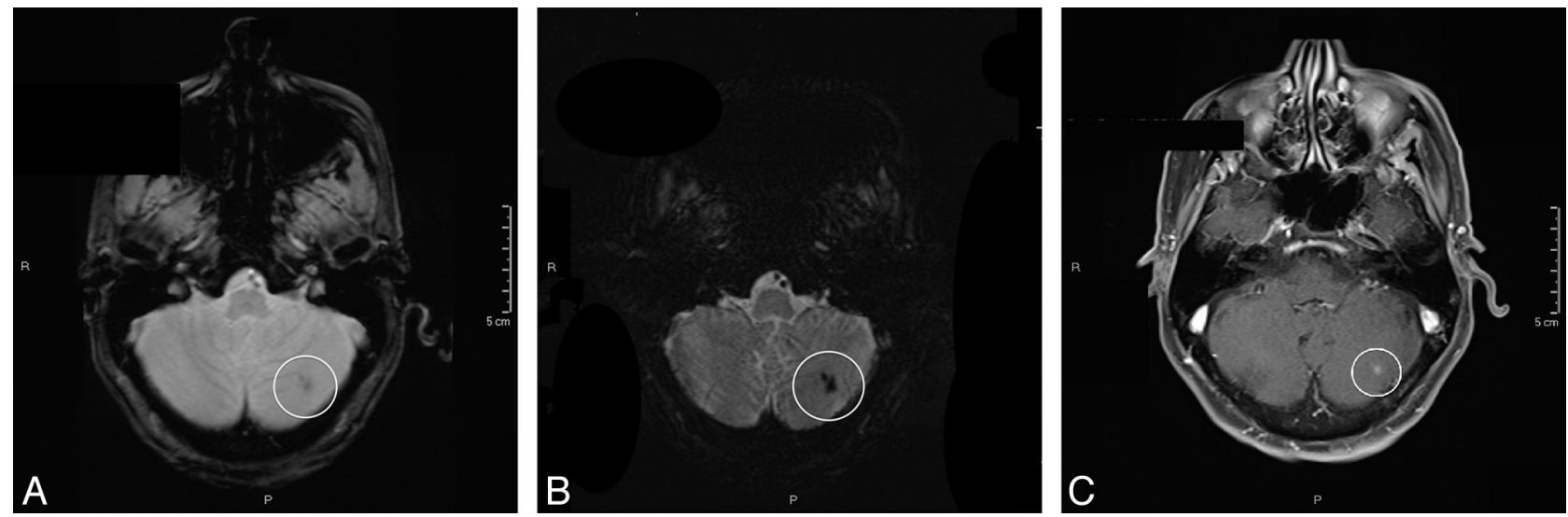

FIG 2. $\mathrm{A} B C T$ (circle) seen more clearly on SWI. A, GRE image; $B$, SWI; $C$, postcontrast $\mathrm{Tl}$ image. 

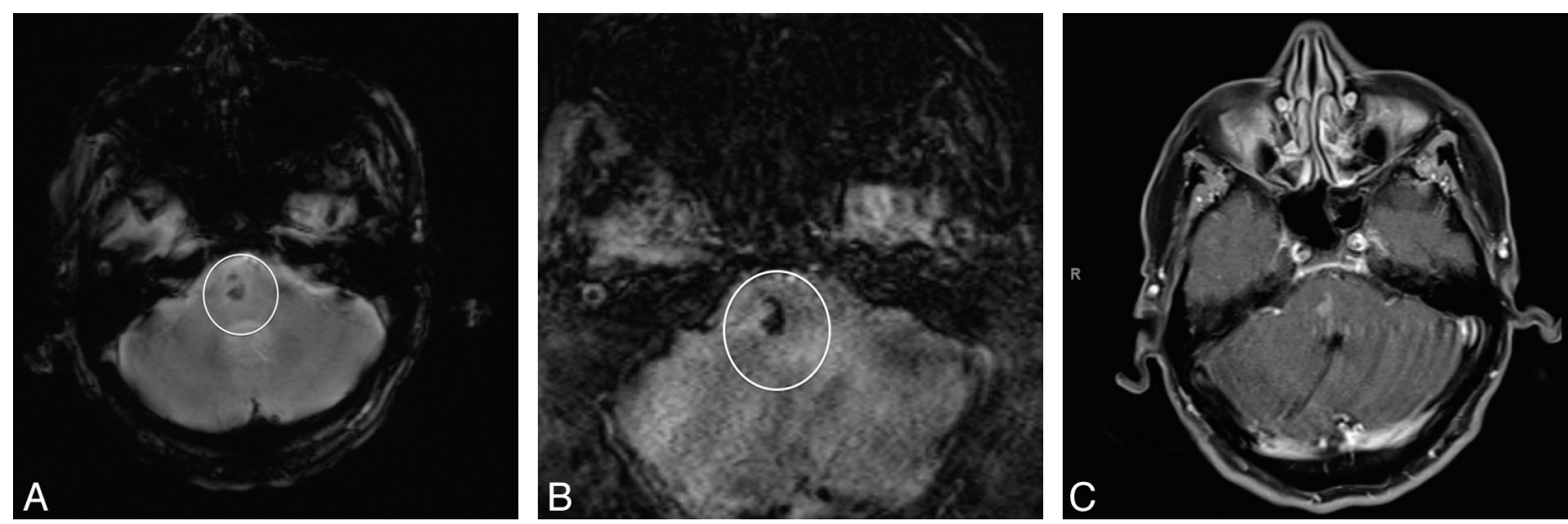

FIG 3. $A B C T$ (circle) seen in the pons on both GRE (A) and SWI $(B)$ and confirmed on postcontrast TI image (C).

versus GRE was statistically significant $\left(z=2.85, P<.01 ; \chi^{2}=\right.$ $8.10, P<.01)$.

\section{DISCUSSION}

BCT is a benign vascular malformation consisting of a cluster of thin-walled capillaries intermixed with normal brain parenchyma, differentiating it from a cavernous malformation, which has no normal brain parenchyma. ${ }^{1,9}$ It is relatively rare, having a reported prevalence of $0.4 \%$, and represents $16 \%-20 \%$ of cerebral vascular malformations. ${ }^{1,9}$ These have a predilection for the pons but have been uncommonly reported in other locations. ${ }^{10}$ Of our 17 cases, 8 (47.0\%) BCTs were in the pons with the others distributed in other locations of the brain (Table 2). Although there are a few case reports of this entity being symptomatic, ${ }^{2}$ BCTs are almost invariably asymptomatic. ${ }^{10}$ With improvement in MR imaging field strength, the detection of these slow-flowing lesions is increasing, creating an increasing dilemma for differentiating them from other postcontrast enhancing lesions such as demyelination, subacute infarction, and neoplasm. ${ }^{1,9}$

Susceptibility in MR imaging is defined as the magnetic response of a substance when placed in an external magnetic field. SWI, which was first described in 1997, is a high-resolution 3D gradient-echo MR imaging sequence that uses magnitude and phase information to create an image from $\mathrm{T} 2{ }^{*}$-contrast and phase changes relying on magnetic susceptibility. ${ }^{11}$ BCTs show increased susceptibility effects ${ }^{9}$ and signal loss on SWI in a focal lesion, in which enhancement is highly specific for a BCT. ${ }^{7}$ As these lesions rarely have hemorrhage or calcifications, slow flow and increased quantities of deoxyhemoglobin are thought to be the causes of increased susceptibility. ${ }^{10}$

Finkenzeller et $\mathrm{al}^{5}$ showed that GRE sequences were particularly useful in detecting BCTs. Lee et $\mathrm{al}^{6}$ demonstrated DWI can also be useful for BCT detection. A case report in 2006 by Yoshida et $\mathrm{al}^{12}$ found a BCT that was occult on GRE but showed signal loss on SWI. A retrospective study in 2012 by El-Koussy et al ${ }^{13}$ examined 33 cases of BCT and found that $100 \%$ showed signal loss on SWI whereas only $39 \%$ showed changes on T1 and T2 images. Our series compared frequency of detection of BCT for SWI versus GRE. In our series, we found no examples of BCT where GRE was positive and SWI was negative. BCTs were more conspicuous on SWI than GRE and other precontrast MR imaging. Of the 17 patients whom we studied, only 7 were diagnosed on GRE imag- ing. Our findings suggest that diagnostic certainty has the potential to increase for focal enhancement when SWI is performed instead of GRE alone. This is most likely related to the fact that SWI is more sensitive to the susceptibility effects that BCT is known to cause. ${ }^{10}$ This is because this sequence additionally relies on phase changes. ${ }^{11}$

Our study was done on $1.5 \mathrm{~T}$ scanners and recent studies have clearly showed increased susceptibility with increased strength of magnetic field. ${ }^{14}$ Future studies can focus on higher field strengths, possibly with $3 \mathrm{~T}$ and using a bigger number of patients.

\section{CONCLUSIONS}

BCT is a potential diagnostic dilemma; increased diagnostic certainty can reduce morbidity and unnecessary testing. In our series, we found no examples of BCT where GRE was positive and SWI was negative. BCTs were more conspicuous on SWI than GRE and, in some cases, were picked up when GRE was negative. SWI therefore appears superior to GRE in the detection of BCT and, though still in its early stages, the initial research shows the addition of SWI has the potential to increase the likelihood of diagnosing BCT and decreasing misdiagnosis.

\section{REFERENCES}

1. Nussbaum ES. Vascular malformations of the brain. Minn Med 2013;96:40-43

2. Scaglione C, Salvi F, Riguzzi P, et al. Symptomatic unruptured capillary telangiectasia of the brain stem: report of three cases and review of the literature. J Neurol Neurosurg Psychiatry 2001;71:390-93

3. Barr RM, Dillon WP, Wilson CB. Slow-flow vascular malformations of the pons: capillary telangiectasia? AJNR Am J Neuroradiol 1996;17:71-78

4. Lee RR, Becher MW, Benson ML, et al. Brain capillary telangiectasia: MR imaging appearance and clinicohistopathologic findings. Radiology 1997;205:797-805

5. Finkenzeller T, Fellner FA, Trenkler J, et al. Capillary telangiectasias of the pons. Does diffusion-weighted MR increase diagnostic accuracy. Eur J Radiol 2010;74:112-16

6. Lee BCP, Vo KD, Kido DK, et al. MR high-resolution blood oxygenation level-dependent venography of occult (low-flow) vascular lesions. AJNR Am J Neuroradiol 1999;20:1239-42

7. Sehgal V, Delproposto Z, Haacke EM, et al. Clinical applications of neuroimaging with susceptibility-weighted imaging. J Magn Reson Imaging 2005;22:439-50

8. McNemar Q. Psychological Statistics. 4th ed. New York: Wiley; 1969:54-58 
9. Byrne JV. Cerebrovascular malformations. Eur Radiol 2005;15: $448-52$

10. Castillo M, Morrison T, Shaw JA, et al. MR imaging and histologic features of capillary telangiectasia of the basal ganglia. AJNR Am J Neuroradiol 2001;22:1553-55

11. Dammann P, Barth M, Zhu Y, et al. Susceptibility weighted magnetic resonance imaging of cerebral cavernous malformations: prospects, drawbacks, and first experience at ultra-high field strength (7-Tesla) magnetic resonance imaging. Neurosurg Focus 2010;29:E5
12. Yoshida Y, Terae S, Kudo K, et al. Capillary telangiectasia of the brain stem diagnosed by susceptibility-weighted imaging. J Comput Assist Tomogr 2006;30:980-82

13. El-Koussy M, Schroth G, Gralla G, et al. Susceptibility-weighted MR imaging for diagnosis of capillary telangiectasia of the brain. AJNR Am J Neuroradiol 2012;33:715-20

14. Campbell PG, Jabbour P, Yadla S, et al. Emerging clinical imaging techniques for cerebral cavernous malformations: a systematic review. Neurosurg Focus 2010;29:E6 